\title{
Letter: Association of Z-Score of the Log-Transformed A Body Shape Index with Cardiovascular Disease in People Who Are Obese but Metabolically Healthy: The Korea National Health and Nutrition Examination Survey 2007-2010 (J Obes Metab Syndr 2018;27:158-65)
}

Eun-Jung Rhee* Department of Endocrinology and Metabolism, Kangbuk Samsung Hospital, Sungkyunkwan University School of Medicine, Seoul, Korea

Obesity is defined as a state of excessive body fat accumulation. Recent studies have suggested that not only the amount of body fat accumulated but also where fat is accumulated matters in the development of comorbidities of obesity. ${ }^{1}$ Although numerous obesity indices to measure body fat have been developed and validated, there is no single index that accurately reflects fat accumulation status in our body that influences cardiometabolic risk. ${ }^{2}$ Body mass index has traditionally been used as the gold standard for the measurement and definition of obesity, but controversy remains due to its failure to reflect abdominal obesity and muscle mass. ${ }^{3,4}$

In this issue of Journal of Obesity \& Metabolic Syndrome, Chung et $\mathrm{al} .{ }^{5}$ analyzed the association of Z-score of the log-transformed A Body Shape Index (LBSIZ), the modified form of A Body Shape Index (ABSI) developed by Krakauer and Krakauer ${ }^{6}$, which is a new equation for estimating body shape using waist circumference (WC), body weight, and height, with cardiovascular disease (CVD) in the metabolically healthy obese ( $\mathrm{MHO}$ ) using the Korea National Health and Nutrition Examination Survey (KNHANES) 20072010. In this study, increasing LBSIZ showed positive correlations with CVD risk in $\mathrm{MHO}$, metabolically unhealthy non-obese, and metabolically unhealthy obese participants compared with metabolically healthy non-obese participants.

Although the results were novel, the design of the study is somewhat complicated in that they not only looked at CVD risk according to LBSIZ, but also expanded the results across $\mathrm{MHO}$ phenotypes, which made the interpretation of the results difficult for readers. Dividing the study into two papers (that is, one that analyzes the CVD risk according to LBSIZ tertiles, and another analyzing the association of LBSIZ across MHO phenotypes) would have improved the interpretation of LBSIZ implications. In addition, it was unclear whether the authors made any attempt to create a new equation for ABSI using KNHANES data, as Krakauer and 
Krakauer $^{6}$ did. A new equation using WC, body weight, and height that reflects body fat in Koreans would be very useful and could be applied in many studies. Nevertheless, the study's results are novel and useful for expanding the implications of ABSI and LBSIZ.

\section{CONFLICTS OF INTEREST}

The author declares no conflict of interest.

\section{REFERENCES}

1. Lim S. Ectopic fat assessment focusing on cardiometabolic and renal risk. Endocrinol Metab (Seoul) 2014;29:1-4.

2. Schneider HJ, Glaesmer H, Klotsche J, Böhler S, Lehnert H, Zeiher AM, et al. Accuracy of anthropometric indicators of obesity to predict cardiovascular risk. J Clin Endocrinol Metab 2007;92:589-94.

3. Cho JH, Rhee EJ, Park SE, Kwon H, Jung JH, Han KD, et al.
The risk of myocardial infarction and ischemic stroke according to waist circumference in 21,749,261 Korean adults: a nationwide population-based study. Diabetes Metab J 2019;43: 206-21.

4. Park J, Lee ES, Lee DY, Kim J, Park SE, Park CY, et al. Waist circumference as a marker of obesity is more predictive of coronary artery calcification than body mass index in apparently healthy Korean adults: the Kangbuk Samsung Health Study. Endocrinol Metab (Seoul) 2016;31:559-66.

5. Chung W, Park JH, Ryu OH, Yu JM, Yoo HJ, Moon S. Association of Z-score of the log-transformed A Body Shape Index with cardiovascular disease in people who are obese but metabolically healthy: the Korea National Health and Nutrition Examination Survey 2007-2010. J Obes Metab Syndr 2018; 27:158-65.

6. Krakauer NY, Krakauer JC. A new body shape index predicts mortality hazard independently of body mass index. PLoS One 2012;7:e39504. 